\title{
Gene expression profiles of some prognostic markers in a human breast cell line (MCF7) exposed to Curcumin nanoparticles and nanocapsules
}

\author{
Emad S. Shaker ${ }^{*}$, Ghada M. Nasr ${ }^{2}$ and Mahmoud Moawad ${ }^{3}$ \\ ${ }^{1}$ Agricultural Biochemistry Dept., Minia University, Egypt., ${ }^{2}$ Department of Molecular Diagnostics, \\ Genetic Engineering, and Biotechnology Research Institute, University of Sadat City, Egypt. ${ }^{3}$ \\ Department of Pathology, National Cancer Institute, Cairo University, Egypt \\ "Corresponding email; emad.shaker@mu.edu.eg
}

\section{ABSTRACT}

Introduction: Worldwide, cancer is a significant public health problem. Curcumin exhibits anti-inflammatory, antiproliferative, and anticancer properties when used in medicine. Investigated study for Curcumin's chemopreventive mechanism against human malignancies, this research examined the cellular and molecular alterations generated by curcumin modified compound in breast cancer (MCF-7) cell lines. Oncogenic EGFR and VEGFR2 mutations lead to the formation, invasion, and maintenance of malignant phenotypes in humans, including breast cancer. Studied prognostic markers such as Cmyc and Ki67 in breast cancer, and the apoptotic gene as Caspase-3 have been done.

Aim of the work: The purpose of this study is to determine the therapeutic efficacy of curcumin nanoparticles and nanocapsules in breast cancer cell lines (MCF7).

Materials and methods: We used real-time PCR to assess the expression of the C-myc, Ki67, EGFR, VEGFR2, and Caspase-3 genes in MCF7 cells treated with Curcumin nanoparticles and nanocapsules.

Results: Curcumin nanoparticles and nanocapsules boosted apoptotic cell populations considerably regardless of the nanotechnology used. Additionally, the mRNA expression analysis results indicated that the mechanism activated by curcumin nanocapsules involved the upregulation of the oncogenes EGFR and VEGFR2. In comparison to curcumin nanoparticles, curcumin nanocapsules significantly reduced the expression of Ki67 and c-myc mRNAs in breast cancer cells. The mRNA expression study revealed that curcumin nanocapsules produce an increase in the apoptotic Caspase-3 gene production compared to cells treated with curcumin nanoparticles.

Conclusion: This work demonstrates that curcumin nanoparticles created using a novel mechanical process can be employed successfully as an anticancer agent. These findings add to our understanding of the molecular mechanisms behind curcumin nanocapsules' anticancer activity in breast cancer.

Keywords: Breast cancer, MCF7 cell line, C-myc, Ki67, EGFR, VEGFR2, Caspase3, gene expression, Curcumin nanocapsules. 


\section{Introduction}

For millennia, cancer has posed a significant threat to public health, and its prevalence is increasing at an alarming rate. According to worldwide cancer statistics (GLOBOCAN) 2020, cancer incidence will grow by around 47\% in 2040 compared to predicted cases in 2020. The increasing number of cancer cases will almost certainly increase the death rate of cancer patients, particularly breast and colorectal cancer [1]. Breast cancer is the second most prevalent malignancy and the sixth most lethal. Breast cancer accounts for $23 \%$ of all female cancer diagnoses and $14 \%$ of cancer deaths. Breast cancer is a term that refers to a group of cancers that arise from the breast epithelial cells. Cell lines can be utilized as in vitro models for cancer research in molecular diagnostics [2].

The EGF receptor (EGFR) controls cell growth, including HER-1, EGFR-2/HER-2, HER-3, and HER-4 (Erb-4). EGFR-1 and HER-2 are overexpressed in breast cancer [3]. The most common EGFR-1 ligands are EGF and TGF, which form complexes with HER2 and other ErbB family receptors. Binding and dimerization activate the intracellular tyrosine kinase [4]. Activated EGFR (pEGFR) promotes MAPK/ERK and PI3K/Akt signaling pathways, causing uncontrolled cell proliferation and apoptosis suppression. Other prognostic factors include the activated EGFR variant (pEGFR) [5]. EGFR phosphorylation has been associated to a poor prognosis in non-small cell lung cancer [6]. Oncogenic EGFR overexpression requires medicines that disrupt EGFR signaling through phosphorylation and its target molecules.

sVEGFR-1 is a soluble receptor tyrosine kinase (RTK) that functions as a negative regulator of VEGF [7]. VEGFR-2 is a VEGF/VEGFR angiogenic signaling receptor. Breast cancer must form localized tumors and spread [8]. Microvessel counts relate higher VEGF expression to poor prognosis in several malignancies, including breast [9].

VEGF controls cancer angiogenesis and cell growth [10]. However, clinical trials have shown that additional inhibitors may be required to control tumor development and proliferation. They employ the same downstream signaling molecules. Tumors with constitutive VEGF overexpression acquire resistance to anti-EGF/EGFR treatment [11]. Activating EGFR signaling increases VEGF production. Because both routes are activing at the same time, combining $\operatorname{EGF}(\mathrm{R})$ and $\operatorname{VEGF}(\mathrm{R})$ inhibition may have anticancer effects. Combining EGFR and VEGFR inhibitors seems to slow tumor development [12]. In colorectal, breast, and lung cancer trials, bevacizumab (humanised anti-VEGF antibody) showed promise [13].

The determination of the proliferative fraction of cancer cells using Ki-67 expression was a simple, reproducible procedure. The expression of Ki-67 was examined in several different types of human cancer. Ki-67 was favorably linked with tumor grade in breast cancer [14]. MYC proteins are transcription factors that regulate gene expression and cancer. Myc family member overexpression is expected in over half of all human cancers. C-myc is the family's defining member, overexpressed in hematologic and solid tumors [15]. 
Caspase 3, Bax, and Bc12 regulate apoptotic cell death and proliferation [16]. Caspase3 is an endoprotease that controls apoptosis and inflammation. Apoptotic executioner caspase- 3 coordinates the destruction of cellular structures such as DNA fragmentation and protein cytoskeletal disintegration. [17].

Nanomedicine is a rapidly growing science that can significantly alter human health [18]. Our group studied curcumin micro and nanocapsules in generated considerable investigation as antioxidant and anticancer agents $[\mathrm{X}, 20]$. Curcumin has gained increased interest for its antibacterial, antioxidant, and anti-aging properties, and its ability to scavenge free radicals. Numerous further researches have credited Curcumin's broad spectrum of therapeutic qualities, [21], including its ability to inhibit cancer cell proliferation, promote tumor cell death, and prevent metastasis [22]. Curcumin was proved to decrease the proliferation of cancer cells in various cancer types, including gastric, hepatic, breast, colon, cervical, and lung cancer [23]. Additionally, curcumin administration significantly alleviates the adverse effects associated with traditional therapy [24].

This study was designed to determine the therapeutic effect of curcumin nanoparticles (CNP) and Curcumin nanocapsules (CNC) on a breast cancer cell line (MCF7) as well as the expression patterns of the EGFR, VEGFR2, C-myc, Ki 67, and caspase 3 genes.

\section{3- Material and Methods}

\section{Preparation of curcumin nanocapsules}

Curcumin nanocapsules CNC were made by mechanically milling (Model: PQ-N2 Planetary Ball Mill, Gear Drive 4-station planetary Ball mill, 220v) [25] at 40,000 rpm for 90 minutes. A magnetic stirrer was employed to extract pulverized Curcuma powder with $95 \%$ ethanol [26]. The CNC manufacture used homogenization, sodium alginate and Tween 20 (T20) matrix [19].

\section{Transmission Electron Microscopy TEM:}

CNC was analyzed using TEM to determine its morphology. After accelerating the voltage and observing the specimens under a microscope, the diameter of the specimens was estimated using micrographs [27].

\section{Molecular Studies}

In breast cancer cell lines [MCF7], we examined the mRNA expression of the EGFR and VEGFR2 genes in combination with B-actin as a housekeeping gene (control gene) implicated in the pathway. The RNA was isolated from the cells using an RNA extraction kit (Quigene). The RNA quality was assessed using agarose gel electrophoresis. NanoDrop 1000 Spectrophotometer assessed optical density (A260/A280 ratio) of isolated RNA (Wilmington, DE, USA). In this case, the REVERTA-L RT reagents kit was employed for cDNA conversion. 
The ExicyclerTM96 Bioneer equipment was used to perform real-time PCR using the SYBR Green dye (KapaBiosystems, Inc., Wilmington, MA, USA) (Bioneer Corporation, Daejoen, Korea).

One gram RNA was reverse transcribed using the qScript cDNATM SuperMix according to the manufacturer's instructions.

The resulting cDNA was then subjected to RT-PCR using the primers specified in Table (1) as follows: 5 minutes at $95^{\circ} \mathrm{C}, 40$ cycles of denaturation for 1 minute, annealing for 45 seconds at $60^{\circ} \mathrm{C}$, extension for 30 seconds at $72^{\circ} \mathrm{C}$, and a final extension for 20 seconds at $72^{\circ} \mathrm{C}[28,29,30,31]$.

Table 1: Primers for all examined genes.

\begin{tabular}{|c|l|l|}
\hline \multirow{2}{*}{ Gene } & \multicolumn{2}{|c|}{ Primers sequence (5-3') } \\
\cline { 2 - 3 } & \multicolumn{1}{|c|}{ Forward } & \multicolumn{1}{c|}{ Reverse } \\
\hline VEGFR2 & GAAGCTCCTGAAGATCTGT & GAGGATATTTCGTGCCGCGC \\
\hline EGFR & GGAGCTGCCCATGAGAAAT & ATTGGGACAGCTTGGATCAC \\
\hline C-myc & CCAGGACTGTATGTGGAGCG & CTTGAGGACCAGTGGGCTGT \\
\hline Ki67 & TCCTTTGGTGGGCACCTAAGACCTG & TGATGGTTGAGGTCGTTCCTTGATG \\
\hline Caspase3 & TTTGTTTGTGTGCTTCTGAGCC & ATTCTGTTGCCACCTTTCGG \\
\hline B-actin & CAGATCATGTTTGAGACCTTCAA & GTGGTACGACCAGAGGCATACA \\
\hline
\end{tabular}

\section{Statistical Analysis}

The SPSS statistical software evaluated the data, followed by WallerDuncan ratio [32].

\section{4- RESULTS5}

\section{Characterization of curcumin nanocapsules}

TEM was used to image curcumin nanocapsules (Figure 1). The images of spherical micelles indicated that nanocapsules have a diameter ranging from 7 to $42 \mathrm{~nm}$ [19]. The particle size distribution of curcumin nanoencapsulation was obtained and analyzed numerically. 


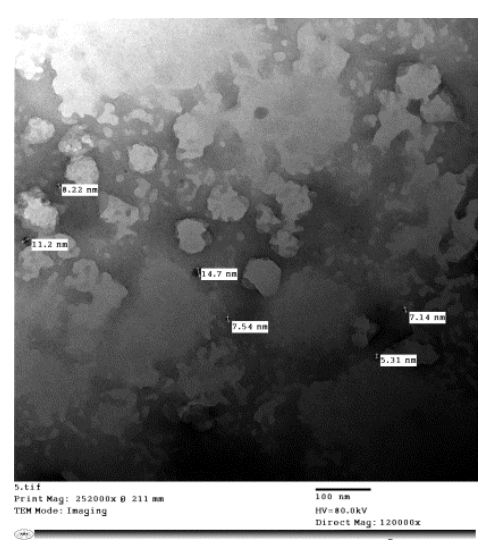

Figure 1. TEM images of Curcumin nanocapsules

\section{Quantitative Real-Time Polymerase Chain Reaction}

The fold change in C-myc, Ki67, EGFR, VEGFR2, and Caspas3 mRNA expression levels in curcumin nanoparticles and nanocapsule-treated MCF7 cells compared to untreated cells is shown in Figures (2, 3, and 4). The present study demonstrated lower expression of the EGFR and VEGFR2 genes in MCF7 cells treated with curcumin nanocapsules compared to MCF7 cells treated with curcumin nanoparticles (figure 2).

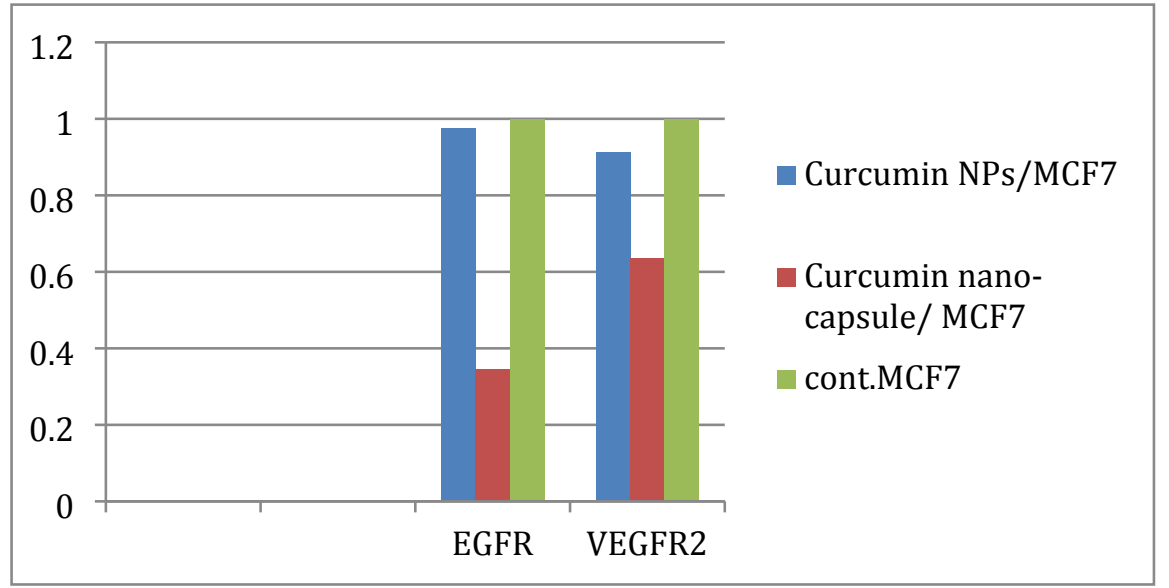

Figure 2. Effect of Curcumin NPs and nanocapsules on VEGFR2 and EGFR genes expression

As shown in Figures, curcumin nanoparticles treated with human breast cancer cells (MCF-7) expressed more Ki67 and C-myc mRNA than curcumin nanocapsules treated with human breast cancer cells (MCF-7) (figure 3). The fold change of the apoptotic gene caspase- 3 and the housekeeping gene-actin as a control in MCF-7 cells treated with curcumin nanoparticles and curcumin nanocapsules compared to untreated cells is shown in Figure (4). When cells treated with curcumin nanoparticles or curcumin nanocapsules were compared to controls, the expression of the caspas3 gene was 
enhanced. On the other hand, the expression of the caspase 3 gene rose considerably following treatment with curcumin nanoparticles. In control RT-PCR analysis, Curcumin did not affect the levels of $\beta$-actin mRNA in either cell line.

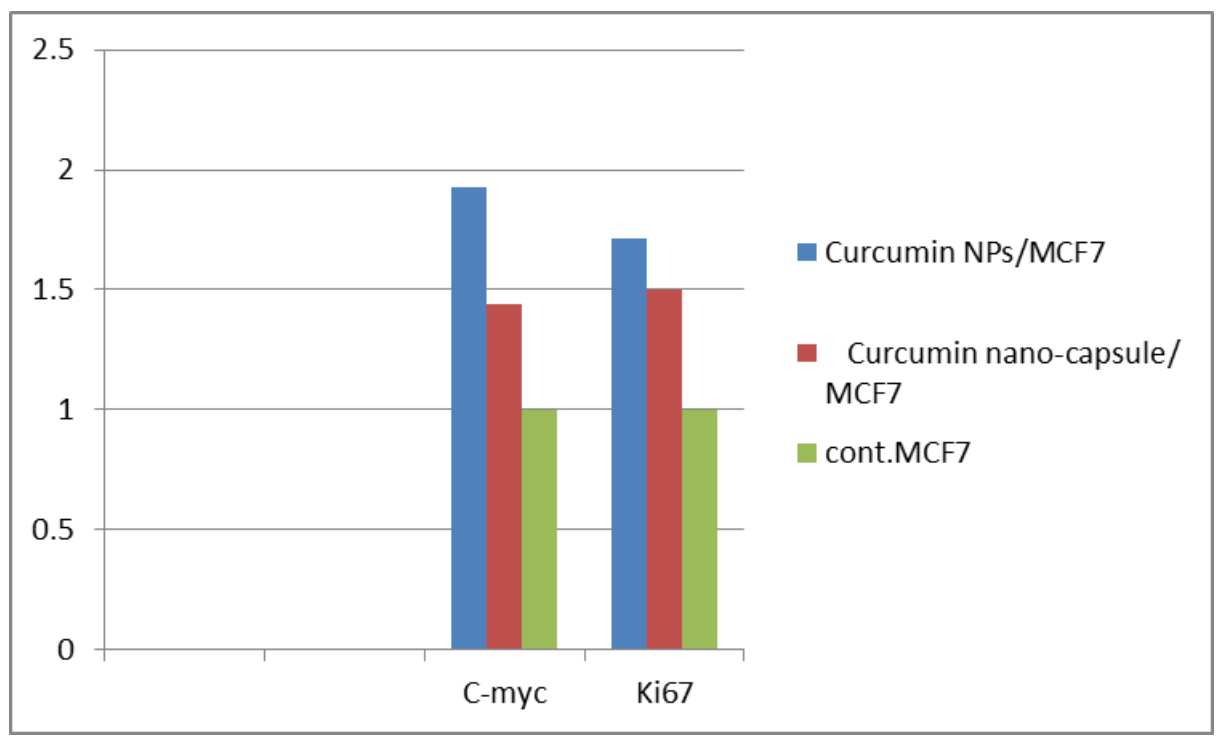

Figure 3. Effect of Curcumin NPs and nanocapsules on C-myc and Ki67genes expression

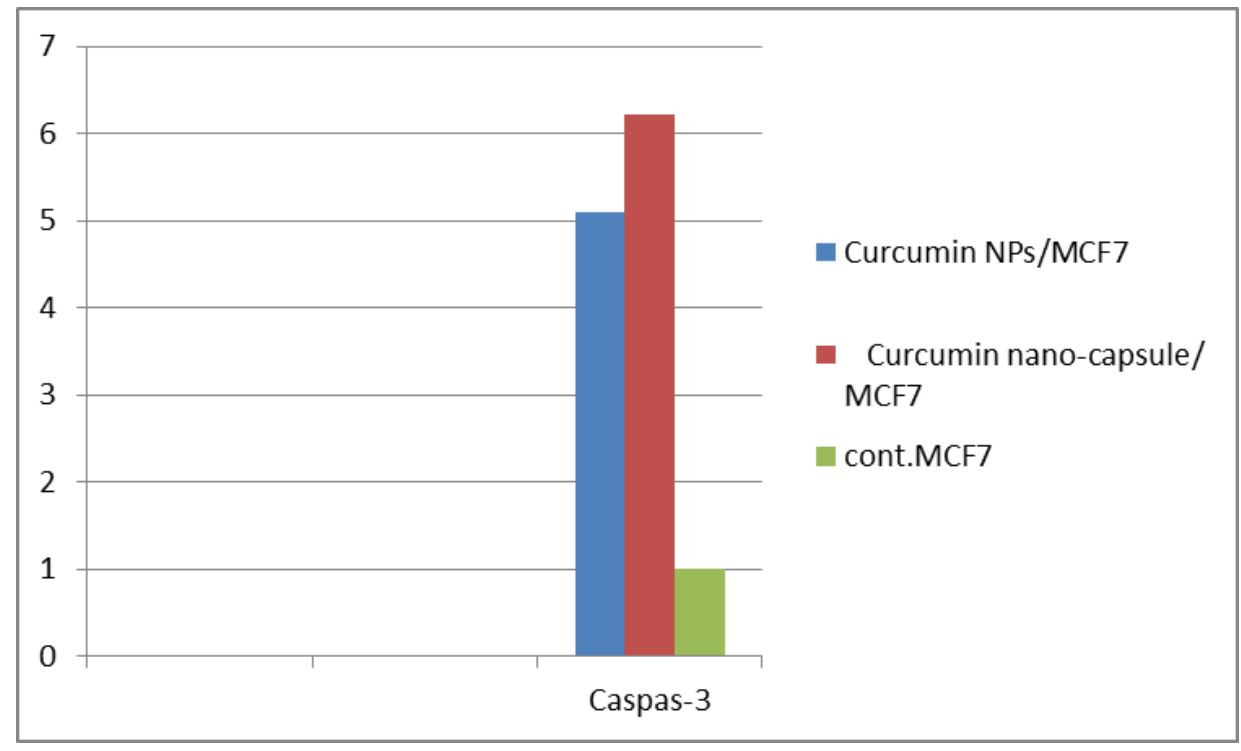

Figure 4. Effect of Curcumin NPs and nanocapsules on Caspase-3 gene expression

\section{5- DISCUSSION}

Locally advanced breast cancer accounts between $30 \%$ and $60 \%$ of cases with breast cancer and gradually will be a clinical challenge because most patients with this diagnosis acquire distant metastases after receiving suitable and effective radiation and 
surgery [33]. Locally advanced breast tumors are frequently accompanied with increased EGF and VEGF expression, decreased relapse-free or overall survival, and disease aggressiveness [34]. Thus, a non-toxic, more effective, and new treatment method is required to combat loco-regional breast cancer recurrence, particularly in individuals treated prior to chemo or radiotherapy.

EGF, VEGF, and their receptors, EGFR and VEGFR, have all been involved in the development of human cancer for an extended time [35]. These variables have solid connections and signaling, resulting in survival and resistance mechanisms that effectively evade targeted therapy [36]. EGFR is a member of the human EGF receptor (HER) family of tyrosine kinases, whose aberrant signaling has been linked in a vast variety of epithelial-derived malignancies, which account for nearly $80 \%$ of all solid tumors. [37]. EGFRs, being one of the most extensively studied receptor tyrosine kinase families, play critical roles in signal transduction and tumorigenesis. Additionally, several cancers exhibit deregulation of the EGFR gene [38]. VEGFR2 receptors were initially assumed to be exclusive to endothelial cells, but recent investigations have revealed that VEGFR2 is widely expressed in human tissues and malignancies, implying an autocrine signaling loop between VEGF and VEGFR2 [39].

Breast cancer is not the only type of cancer that is well-known. Two molecular subtypes have been identified using gene expression profiling, with diagnosis based on the presence or lack of the hormone receptor-related genes EGFR and VEGFR2. Proangiogenic factors are overexpressed in several types of malignancies. MCF7 breast cancer cells, one of the most aggressive kinds of cancer, have been discovered to upregulate EGFR and VEGF/VEGFR expression, both pro-angiogenic factors. The EGFR and VEGFR2 signaling pathways have been found to interact in tumor formation, survival, and angiogenesis. ERK and PI3 K/Akt signaling are activated by EGFR and VEGFR2 [40]. This regulation is shown in cancer cells that express EGFR and VEGFR2 [41]. Because we used human ramucirumab in our xenograft trial, it seems improbable that the ramucirumab arm of anti-EGFR/VEGFR2 monoclonal antibodies inhibited angiogenesis. Anti-EGFR/VEGFR2 BsAbs have anticancer efficacy by blocking EGFR signaling and altering VEGF/VEGFR2-mediated autocrine/paracrine pathways, which is consistent with our results in cellular models. To ascertain the effect of CNPs and CNCs, we analyzed the oncogene repressors EGFR and VEGFR2 to discover the mechanism by which they augment the effect of CNPs and CNC; we also conducted some tests on the expression of genes implicated in the oncogenic pathway.

In contrast to individual exposure, real-time PCR analysis demonstrated that cotreatment with CNP and CNC leads to a significant drop in EGFR and VEGFR2 expression levels in curcumin nanoparticles and nanocapsule treated cells compared to the control untreated cell line. The current study showed that when MCF7 cells were treated with CNPs, EGFR and VEGFR2 expression was increased compared to when MCF7 cells were treated with Curcumin nanocapsules. Our findings are consistent with those reported by Sarkar et al. [42]. 
The proto-oncogene c-myc is known to influence tumor formation and apoptotic cell death. A previous study indicated a link between p53 mutations and c-myc activation [43]. Ki67 was first identified in 1983 as a nuclear protein in a Hodgkin's lymphoma cell line. The expression of Ki-67 was examined in some different types of human cancer. Ki67 had a positive correlation with breast cancer grade [44].

Intracellular caspases, a class of structurally related cysteine proteases discovered previously [45], are key signaling channels involved in apoptotic cell death. Caspase activity is involved in the cleavage of cellular proteins that are often proteolyzed during apoptosis, either directly or indirectly. Caspases $-2,-3,-6,-7$, and -9 , for example, can cleave poly (ADP ribose) polymerase (PARP) [46]. Curcumin was reported to boost the expression of downstream cleaved caspase 3, indicating that it may stimulate the mitochondrial apoptotic pathway [47].

Curcumin is a highly effective anticancer drug that acts differently on different cells. Curcumin's capacity in causing apoptosis for various cancer cells, made scientists expect that one day curcumin derivatives be developed as universal anticancer treatment. Curcumin, a naturally occurring polyphenol chemical derived from the annual herb Curcuma longa, has been demonstrated to effectively treat malignant and benign tumors, inflammation, and a variety of other ailments [48].

This work was designed to examine the molecular mechanisms underlying the EGFR, VEGFR2, C-myc, KI67, and caspase3 gene pathways in a human breast cancer cell line (MCF7) treated with curcumin nanoparticles and nanocapsules. Scientists can create microscopic particles using nanotechnology. When nanoparticles are smaller than $20 \mathrm{~nm}$ or $50 \mathrm{~nm}$, they can easily pass through blood vessel walls and even into most body cells, making them an attractive candidate for specialized pharmaceuticals employed in the targeted delivery of large doses of chemotherapeutic agents [49]. TEM was used to image curcumin nanocapsules. The images of spherical micelles indicated that nanocapsules have a diameter ranging from 7 to $42 \mathrm{~nm}[19,50]$ and described free curcumin powder as having an irregular form and a mean particle size of around $3.58 \mathrm{~m}$. Our research found that mechanical nano preparation reduced the size of curcumin powder by around 100 times, enhancing the drug's anticipated anticancer efficacy. Curcumin nanocapsules had a smooth surface and a spherical shape, comparable to those discovered in a previous study for curcumin nanoparticles [51].

To better understand the molecular alterations generated by curcumin administration, we used RT-PCR to examine the expression of Ki67, C-myc, and caspase 3 mRNA. In breast cancer cells, curcumin nanoparticles and nanocapsules treatment boosted the expression of Ki67, C-myc, and Caspas 3 mRNAs, compared to untreated control cells (figures 3, and 4). In MCF-7 cells, treatment with curcumin nanocapsules decreased Ki67 and C-myc mRNA levels while increasing up-regulation compared to treatment with curcumin nanoparticles. Our findings corroborate previous findings $[52,53]$. Finally, it can be stated that Curcumin nanoparticles synthesized using novel mechanical processes can be used successfully as anticancer drugs. These findings 
add to our understanding of the molecular mechanisms behind curcumin nanocapsules' anticancer activity in breast cancer cells.

\section{6- REFERENCES}

1. Sung H, Ferlay J, Siegel RL, Laversanne M, Soerjomataram I, Jemal A and Bray F. Global cancer statistics 2020; GLO-BOCAN Estimates of incidence and mortality worldwide for 36 cancers in 185 countries. CA Cancer J. Clin. 2021; 71, 209-249.

2. McMillon E, McKenna W, Milne C. Guidelines on preparing a medical report for compensation purposes. Br. J. Dermatol. 1982; 106, 489-494.

3. Bianco R, Gelardi T, Damiano V, Ciardello F, Tortora G. Rational bases for developing EGFR inhibitors for cancer treatment. Int J Biochem Cell Biol 2007; 39:1416-31.

4. Gohring UJ, Ahr A, Scharl A, Weisner V, Neuhaus W, Crombach G, et al. Immunohistochemical detection of epidermal growth factor receptor lacks prognostic significance for breast carcinoma. J Soc Gynecol Invest 1995; 2:653-9.

5. Arteaga CL, Baselga J. Clinical trial design and endpoints for epidermal growth factor receptor-targeted therapies: implications for drug development and practice. Clin Cancer Res 2003; 9:1579-89.

6. Kanematsu T, Yano S, Uehara H, Bando Y, Sone S. Phosphorylation, but not overexpression, of epidermal growth factor receptor is associated with poor prognosis of non-small cell lung cancer patients. Oncol Res 2003; 13:289-98.

7. Bando H. Vascular endothelial growth factor and bevacizumab in breast cancer. Breast Cancer 2007; 14:163- 73.

8. Folkman J. Tumor angiogenesis: therapeutic implications. N Engl J Med 1971; 285:1182-6.

9. Meunier-Carpentier S, Dales JP, Djemli JP, Garcia S, Bonnier P, Andrac-Meyer $\mathrm{L}$, et al. comparison of the prognosis indication of VEGFR-1 and VEGFR-2 and Tie2 receptor expression in breast carcinoma. Int J Oncol 2005; 26:977-84.

10. Goel HL, Mercurio AM. VEGF targets the tumor cell. Nat Rev Cancer. 2013; 13(12): 871-882.

11. Viloria-Petit A, Crombet T, Jothy S, Hicklin D, Bohlen P, Schlaeppi JM, et al. Acquired resistance to the antitumor effect of epidermal growth factor receptorblocking antibodies in vivo: a role for altered tumor angiogenesis. Cancer Res 2001; 61:5090-101.

12. Emlet DR, Brown KA, Kociban DL, Pollice AA, Smith CA, Ong BB, et al. Response to trastuzumab, erlotinib and bevacizumab, alone and in combination, is correlated with the level of human epidermal growth factor receptor-2 expression in human breast cancer cell lines. Mol Cancer Ther 2007; 6:2664-74.

13. Miller KD, Chap LI, Holmes FA, Cobleigh MA, Marcom PK, Fehrenbacher L, et al. Randomized phase III trial of capecitabine compared with bevacizumab plus capecitabine in patients with previously treated metastatic breast cancer. J Clin Oncol 2005; 23:792- 9. 
14. G. Gasparini S, Dal Fior F, Pozza P, Bevilacqua. Correlation of growth fraction by Ki-67 immunohistochemistry with histologic factors and hormone receptors in operable breast carcinoma, breast. Cancer. Res. Treat. 14: 1989; 329-336.

15. Lance RT and William PT. MYC and Chromatin. The Open Access Journal of Science and Technology. 2015; Vol. 3, Article, ID 101124, 26 pages.

16. Riedl SJ and Shi Y. Molecular mechanisms of caspase regulation during apoptosis. Nat Rev Mol Cell Biol. 2004; 5:897-907.

17. McIlwain DR, Berger T, Mak TW. Caspase functions in cell death and disease. Cold Spring Harb Perspect Biol. 2013; Apr 1;5(4):a008656. Erratum in: Cold Spring Harb Perspect Biol. 2015; Apr;7(4). pii: a026716.

18. Teli MK, Mutalik S, Rajanikant GK. Nanotechnology and nanomedicine: going small means aiming big. Curr Pharm Des. 2010; 16(16):1882-1892.

19. Shaker, E., Fawzy, M., Fahmy, Kh., Moawad, M. and El-Shafei, M. (2022) Nanocapsulation of curcumin and its protective effects against oxidative stress and carcinoma HepG2, MCF7 cells. Egy J Chem (under press)

20. Tomeh MA, Hadianamrei R, Zhao X. A Review of Curcumin and Its Derivatives as Anticancer Agents. Int. J. Mol. Sci. 2019; 20, 1033.

21. Gupta SC, Patchva S, Aggarwal BB. Therapeutic Roles of Curcumin: Lessons Learned from Clinical Trials. AAPS J. 2012; 15, 195-218.

22. Kunnumakkara AB, Bordoloi D, Padmavathi G, Monisha J, Roy N, Prasad S, Aggarwal BB. Curcumin, the golden nutraceutical: Multitargeting for multiple chronic diseases. Br. J. Pharmacol. 2016, 174, 1325-1348.

23. Vishvakarma NK. Novel antitumor mechanisms of Curcumin: implication of altered tumor metabolism, reconstituted tumor microenvironment and augmented myelopoiesis . Phytochem Rev. 2014; 13:717-724.

24. Peng M, Xiao D, Bu Y, Long J, Yang X, Lv S, Yang X. Novel Combination Therapies for the Treatment of Bladder Cancer. Front. Oncol.2021, 10, 3163.

25. Loh ZH, Samanta AK and Heng PWS. "Overview of milling techniques for improving the solubility of poorly water-soluble drugs." Asian journal of pharmaceutical sciences. 2015; 10(4): 255-274.

26. Terjung N, Löffler M, Gibis M, Hinrichs J and Weiss J. "Influence of droplet size on the efficacy of oil-in-water emulsions loaded with phenolic antimicrobials." Food \& function. 2012; 3(3): 290-301.

27. Abd-Elsalam KA and Khokhlov AR. "Eugenol oil nanoemulsion: antifungal activity against Fusarium oxysporum f. sp. vasinfectum and phytotoxicity on cottonseeds." Applied Nanoscience. 2015; 5(2): 255-265.

28. Korashy HM, Maayah ZH, Al anazi FE, Alsaad AM, Alanazi IO, Belali OM, Alatawi FO and Alshamsan AWS. Sunitinib inhibits breast cancer cell proliferation by inducing apoptosis, cell-cycle arrest and dna repair while inhibiting nf-kb signaling pathways; anticancer research. 2017; 37: 4899-4909

29. Saryeddine L, Zibara K, Kassem N, Badran B, El-Zein N. EGF-Induced VEGF Exerts a PI3K-Dependent Positive Feedback on ERK and AKT through VEGFR2 in Hematological In Vitro Models. PLOS ONE journal. 2016; pone.0165876, 2.

30. Pillai GR, Srivastava AS, Hassanein TI, Dharam P. Chauhan DP, Carrier E. Induction of apoptosis in human lung cancer cells by Curcumin. Cancer Letters 208. 2004; 163-170 
31. Potemski P, Pluciennik E, Bednarekc AK, Kusinsk R, Kubiak R, JesionekKupnicka D, Watalad C, Kordek R. Ki-67 expression in operable breast cancer: A comparative study of immunostaining and a real-time RT-PCR assay. Pathology Research and Practice 202. 2006; 491-495

32. Waller RA, \& Duncan DBA. Bayes rule for the symmetric multiple comparisons problem. Journal of the American Statistical Association, 1969; 64, 1484e1503.

33. Rustogi A, Budrukkar A, Dinshaw K, Jalali R. Management of locally advanced breast cancer: evolution and current practice. J Cancer Res T. 2005; 1:21-30.

34. Foekens JA, Peters HA, Grebenchtchikov N, Look MP, Meijer-van Gelder ME, Geurts-Moespot A, van der Kwast TH, Sweep CG, Klijn JG. High tumor levels of vascular endothelial growth factor predict poor response to systemic therapy in advanced breast cancer. Cancer res. 2001; 61:5407-5414.

35. Citri A and Yarden Y. EGF $\pm \mathrm{ERBB}$ signalling: towards the systems levels. Nat Rev Mol Cell Biol. 2006; 7(7): 505 5516.

36. Lee SH, Jeong D, Han YS, Baek MJ. Pivotal role of vascular endothelial growth factor pathway in tumor angiogenesis. Ann Surg Treat Res. 2015; 89(1): $1 \pm 8$.

37. Tan CS, Cho BC, Soo RA. Next-generation epidermal growth factor receptor tyrosine kinase inhibitors in epidermal growth factor receptor -mutant non-small

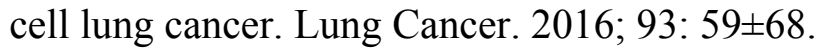

38. Roskoski R Jr. Small molecule inhibitors targeting the EGFR/ErbBfamily of protein-tyrosine kinases in human cancers. Pharmacol Res. 2019; 139: 395- 411.

39. Stewart M, Turley H, Cook N, Pezzella F, Pillai G, Ogilvie D, Cartlidge S, Paterson D, Copley C, Kendrew J, Barnes C, Harris AL, Gatter KC. The angiogenic receptor KDR is widely distributed in human tissues and tumours and relocates intracellularly on phosphorylation. An immunohistochemical study, Histopathology, 2003; 43(1):33-39.

40. Wee P, Wang Z. Epidermal Growth Factor Receptor Cell Proliferation Signaling Pathways. Cancers. 2017; 9, 52.

41. Chen Z, Xie W, Acheampong DO, Xu M, He H, Yang M, Li C, Luo C, Wang M, Zhang JA. Human IgG-like bispecific antibody co-targeting epidermal growth factor receptor and the vascular endothelial growth factor receptor 2 for enhanced antitumor activity. Cancer Biol. Ther. 2016; 17, 139-150.

42. Sarkar S, Mazumdar A, Dash R, Sarkar D, Fisher PB \& Mandal M. ZD6474, a dual tyrosine kinase inhibitor of EGFR and VEGFR-2, inhibits MAPK/ERK and AKT/PI3-K and induces apoptosis in breast cancer cells, Cancer Biology \& Therapy. 2010; 9:8, 592-603.

43. Ragimov N, Krauskopf A, Navot N, Rotter V, Oren M, Aloni Y. Wild-type but not mutant p53 can repress transcription initiation in vitro by interfering with the binding of basal transcription factors to the TATA motif, Oncogene 8. 1993; 1183-1193.

44. Gasparini G, Dal Fior S, Pozza F, Bevilacqua P. Correlation of growth fraction by Ki-67 immunohistochemistry with histologic factors and hormone receptors in operable breast carcinoma, breast. Cancer. Res. Treat. 14. 1989; 329-336.

45. Salvesen GS, Dixit VM. Caspases: intracellular signaling by proteolysis, Cell 91. 1997; 443-446.

46. Nagata S. Apoptosis by death factor, Cell 88. 1997; 355-365. 
47. Hu S, Xu Y, Meng L, Huang L, Sun H. Curcumin inhibits proliferation and promotes apoptosis of breast cancer cells. Exp Ther Med. 2018; 16(2):1266-1272.

48. Sandur SK, Ichikawa H, Pandey MK, et al. Role of pro-oxidants and antioxidants in the anti-inflammatory and apoptotic effects of Curcumin (diferuloylmethane). Free Radic Biol Med. 2007; 43:568-80.

49. Rahimi HR, Nedaeinia R, Shamloo AS, Nikdoust S. Oskuee RK. Novel delivery system for natural products: Nanocurcumin formulations. Avicenna Journal of Phytomedicine. 2016; 6(4):383-398.

50. Zhou X, Su J, Feng S, Wang L, Yin X, Yan J and Wang Z. Antitumor activity of Curcumin is involved in down-regulation of YAP/TAZ expression in pancreatic cancer cells. Oncotarget 7. 2016; 79076-79088.

51. Chuacharoen $\mathrm{T}$ and Sabliov CM. Comparative effects of curcumin when delivered in a nanoemulsion or nanoparticle form for food applications: Study on stability and lipid oxidation inhibition. LWT-Food Science and Technology. 2019; 108319.

52. Gartenhaus RB, Wang P, Hoffmann P. Induction of the WAF/CIP1 protein and apoptosis in human T-cell leukemia virus type I-transformed lymphocytes after treatment with adriamycin by using a p53-independent pathway. Proc Natl Acad Sci USA. 1995; 93: 265-268.

53. Wu GS, El-Deiry WS. Apoptotic death of tumor cells correlates with chemosensitivity independent of p53 or Bcl-2. Clin Cancer Res. 1996; 2: 623633. 